\title{
Design Optimization of the Lines of the Bulbous Bow of a Hull Based on Parametric Modeling and Computational Fluid Dynamics Calculation
}

\author{
Weilin Luo ${ }^{1,2, *}$ and Linqiang Lan ${ }^{1}$ \\ 1 School of Mechanical Engineering and Automation, Fuzhou University, Fuzhou 350116, China; \\ lanlinqiang@hotmail.com \\ 2 Fujian Province Key Laboratory of Structural Performances in Ship and Ocean Engineering, \\ Fuzhou 350116, China \\ * Correspondence: wlluo@fzu.edu.cn; Tel.: +86-591-228-64791 \\ Academic Editor: Fazal M. Mahomed \\ Received: 15 August 2016; Accepted: 23 December 2016; Published: 31 December 2016
}

\begin{abstract}
To reduce the ship wave-making resistance, the lines of the bulbous bow of a hull are optimized by an automatic optimization platform at the ship design stage. Parametric modeling was applied to the hull by using non-uniform rational basis spline (NURBS). The Rankine-source panel method was used to calculate the wave-making resistance. A hybrid optimization strategy was applied to achieve the optimization goal. A Ro-Ro ship was taken as an example to illustrate the optimization method adopted, with the objective to minimize the wave-making resistance. The optimization results show that wave-making resistance obviously reduces and the wave-shape of the near bow becomes gentle after the lines of the bulbous bow of the hull are optimized, which demonstrates the validity of the proposed optimization design strategy.
\end{abstract}

Keywords: hull forms optimization; parametric modeling; computational fluid dynamics calculation; wave-making resistance

\section{Introduction}

Design of ship hull forms is one of the most important contents of the general ship design. How to reduce the ship resistance by means of well-designed forms is always the concern for ship designers and/or researchers. The parent ship based design method predominates in the field of ship design, by which the designed hull can inherit some excellent performance from the parent ship. For a long time, designer's experience played an important role in obtaining the desired hull forms. With the development of computer science and technology, the quality of ship design has been improved considerably by introducing some computational numerical calculation methods. For example, computational fluid dynamics (CFD) based numerical simulation can be used to evaluate the hydrodynamic performances of a ship after the hull forms are designed. By modifying the hull forms and repeatedly performing CFD simulation, the satisfactory hull forms can be obtained. To improve the design efficiency, computer aided design (CAD) was proposed to combine with CFD in the optimization design of hull forms [1]. During the past decade, CAD/CFD integrated hull-form design systems have been developed and successfully applied to the drag reduction of ships [2]. For example, Percival et al. combined a simple CFD tool and B-Spline method to the optimization design of a Wigley ship [3]. Yusuke et al. presented the bulbous bow optimization of a container ship based on the naval architectural package (NAPA) system and a Rankine-source panel method [1]. Tahara et al. applied NAPA and a RANS (Reynolds-averaged Navier-Stokes) solver to the optimization design of a container ship [4]. Abt and Harries presented the generic, multi-objective ship design 
optimization based on software of the Ship Design Laboratory of the National Technical University of Athens (NTUA-SDL) in which well-established naval architectural and optimization software packages are integrated [5]. Harries and Abt distinguished three types of coupling of CAD and CFD [6]. Couser et al. explored the multi-dimensional design-space using first-principle methods within the FRIENDSHIP framework [7]. Brizzolara and Vernengo proposed an integration of a parametric generation module, a multi-objective optimization algorithm and a CFD solver in the optimization of a new unmanned surface vehicle (USV) and small waterplane area twin hull (SWATH) [8]. Biliotti et al. presented a fully automatic optimization chain to the optimization of fast naval vessels by adopting the ModeFrontier optimization environment to the FRIENDSHIP framework, the CFD codes and a multi-objective genetic algorithm [9]. Ginnis et al. employed the NURBS (non-uniform rational basis spline) method and Kelvin-source method to the optimization of a container ship [10]. Brenner et al. used the FRIENDSHIP-SHIPFLOW optimization scheme to retrofit the ships in operation [11]. Zakerdoost et al. used the NURBS method and potential theory to the optimization design of a Wigley ship [12]. Vernengo et al. presented the optimization of a Semi-SWATH by a fully parametric model of the unconventional hull form, a 3D linear Rankine-sources panel method, and a multiobjective global convergence genetic algorithm [13]. Combined with the optimization of a high speed round bilge monohull, Brizzolara et al. addressed the significance of parametric hull form definition by comparing it with Free Form Deformation technique [14]. Ang et al. proposed the Free Form Deformation and potential theory to the optimization design of a heavy-lift and pipe-lay vessel [15]. Wang proposed the NURBS method and CFD calculation based on Neumann-Michell theory to the optimization design of several life ships [16].

Besides the parametric modeling of hull forms and calculation of hydrodynamic performances, optimization is another important factor in the hull-form design system. To guarantee the optimum design and the solving efficiency, an appropriate optimization algorithm is vital to the optimizer. During the last several decades, many optimization algorithms have been proposed such as successive quadratic programming (SQP) [17], genetic algorithm (GA) [18], simulated annealing (SA) [19], particle swarm optimization (PSO) [20], infeasibility driven evolutionary algorithm (IDEA) [21], and quasi-Newton method [10], among others. Generally, these algorithms can be categorized into gradient-based and derivative-free methods [22]. For the gradient-based optimization algorithms (e.g., SQP and quasi-Newton approaches), the interest is the fast convergence; however, the limitation is the locally optimal solution. For the derivative-free optimization algorithms (e.g., GA, SA, PSO, and IDEA approaches), the advantage is the global optimization; however, the convergence rate cannot be guaranteed. Since each optimization algorithm has its advantages and disadvantages, a hybrid optimization scheme should be preferable. In this paper, we perform the combination of a global optimization method (Ensemble Investigation) and a local optimization method (T-Search algorithm) to the optimization design of hull forms of a surface ship. The design optimization is carried out in the SHIPFLOW-CAESES (former FRIENDSHIP) DESIGN environment. Based on the automatic optimization platform, the parametric modeling and the CFD calculation are integrated. The NURBS method is employed to the parametric modeling while the Rankine-source panel method is applied to calculate the wave-making resistance. The minimization of the wave-making resistance is taken as the optimization objective while the design variables are form parameters of the lines of the bulbous bow.

The rest of the paper is organized as follows. In Section 2, the parametric modeling method is introduced; in Section 3, the CFD method to calculate the wave-making resistance is described; in Section 4, the hybrid optimization algorithm is addressed; in Section 5, the entire optimization scheme is described; in Section 6, an example is studied to confirm the validity of the optimization strategy proposed in the study; and the final section is the concluding remarks.

\section{Parametric Modeling of Hull Forms}

In the geometry modeling of a hull, it is often required to obtain the forms rapidly. In addition, a large number of forms should be analyzed so that the optimization can be efficiently performed. 
In this paper, the NURBS method is employed to meet the mentioned requirements. Based on this method, the high-quality of the lines can be guaranteed with fairly less design variables than conventional design methodology. This method provides the best way to establish the relationship between parameters and hull forms and makes sure that the design variation among the entire optimization process is effective and feasible. The parametric model is generated by CAESES-Modeler. The process of curves generation starts with a set of given data elements that are used to define the complete parametric curve. Within the modeler, one can define a set of longitudinal lines and express them as basic curves to describe the ship hull by means of differential, integral and topological information. Transversal basic curves are able to be formed as soon as the longitudinal basic curves are defined (by fair B-Spline parametric curves adopted in the study). Then, a series of surfaces can be set up by corresponding transversal sections that stem from the parametric hull model. Generally, the design of the fully parametric hull surfaces makes it possible to transform the hull form efficiently and effectively. Moreover, all of the hull topologies, the hull sections and the whole hull surface can be generated using the basic curves specifically and precisely. Usually, the hull forms are described by feature curves [23], e.g., the design waterline, flat of side, flat of bottom, etc., which are listed in Table 1 and some of the curves are shown in Figure 1.

Table 1. Basic feature curves describing hull forms.

\begin{tabular}{ccc}
\hline Characteristic & Curve & Abbreviation \\
\hline Position & Design waterline & DWL \\
& Deck line & DEC \\
& Flat of side curve & FOS \\
& Flat of bottom curve & FOB \\
Tangent angle & Center plane curve & CPC \\
Curvature & Tangent angle at beginning and end & TAB, TAE \\
Area & Curvature at beginning and end & CAB,CAE \\
Centroid of area & Sectional area curve & SAC \\
& Vertical and Lateral moments of sectional area & VMS, LMS \\
\hline
\end{tabular}

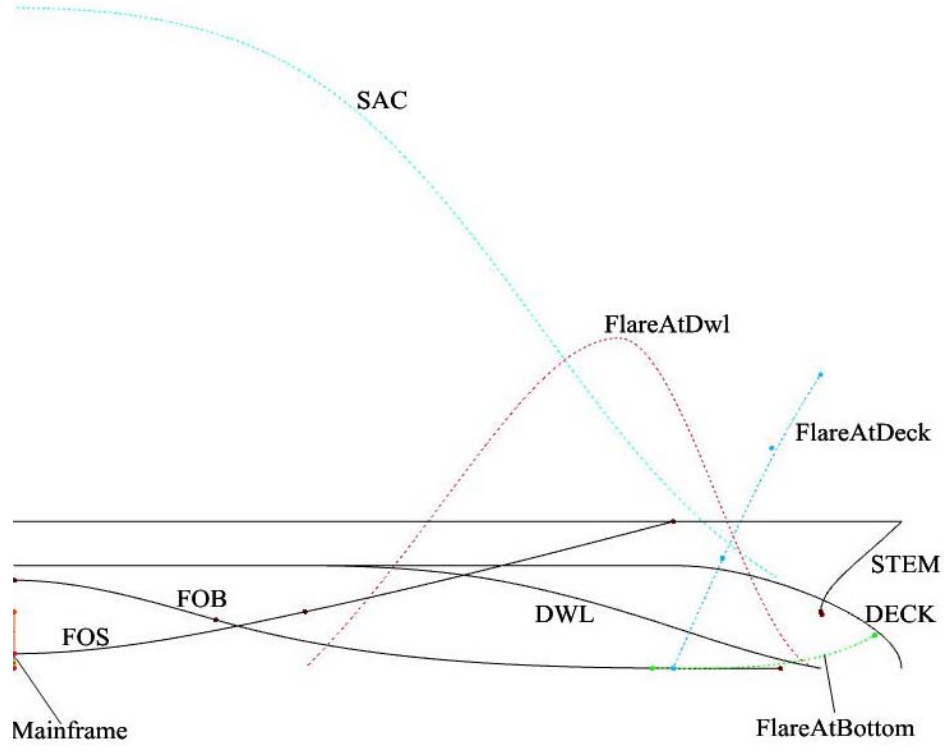

Figure 1. Basic feature curves describing hull forms.

For the bulbous bow, the basic feature curves include: the top longtitudinal section line, the low longtitudinal section line, the half beam line, and the height line of the maximal breadth, as shown in Figure 2. The shape of the bulbous bow can be modified by changing these basic feature curves that 
can be achieved by controlling design variables. Figure 3 presents an example of the variation of the shape of a bulbous bow by modifying these feature curves.

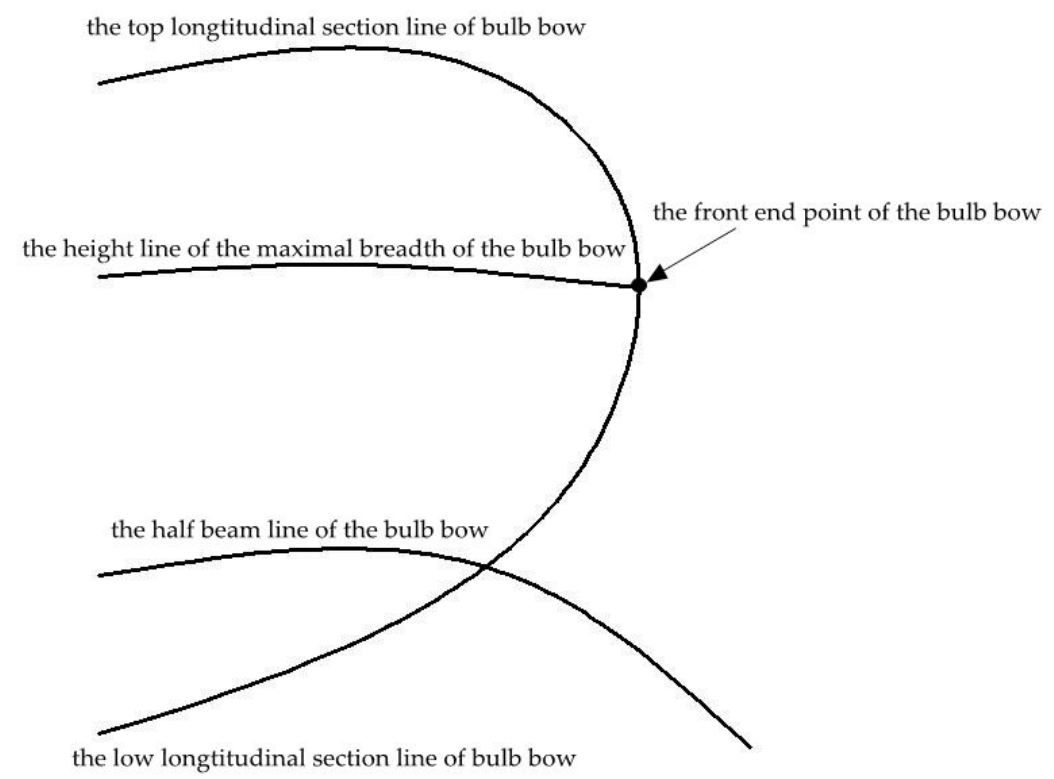

Figure 2. The basic feature curves of a bulbous bow.
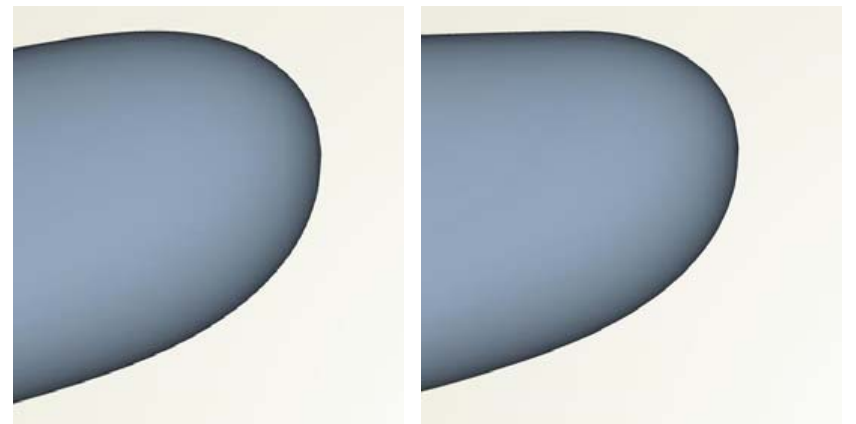

Figure 3. Transformation of a bulb bow: left, plot-original; right, plot-modified.

Using the NURBS method, curves can be expressed as [24]:

$$
Q(t)=\left(\begin{array}{l}
x(t) \\
y(t)
\end{array}\right)=\frac{\sum_{i=0}^{n} w_{i} N_{i, k}(t) d_{i}}{\sum_{i=0}^{n} w_{i} N_{i, k}(t)}, 0 \leq t \leq 1
$$

where $n$ is the number vertex points, $w_{i}$ is the weight, $d_{i}$ is the control point, and $N_{i, k}(t)$ is the B-Spline basis function. For the hull forms, a NURBS surface is a bi-variate vector-valued piecewise rational function as [24]:

$$
S(u, v)=\left(\begin{array}{l}
x(u, v) \\
y(u, v) \\
z(u, v)
\end{array}\right)=\frac{\sum_{i=0}^{n} \sum_{j=0}^{m} N_{i, p}(u) N_{j, q}(v) w_{i, j} P_{i, j}}{\sum_{i=0}^{n} \sum_{j=0}^{m} N_{i, p}(u) N_{j, q}(v) w_{i, j}}, 0 \leq u, v \leq 1
$$

where $P_{i, j}$ forms a bidirectional control net [12]. $N_{i, p}(u)$ and $N_{j, q}(v)$ are B-Spline basis functions in the direction of $\vec{U}=\left\{u_{0}, \cdots, u_{n+p+1}\right\}$ and $\vec{V}=\left\{v_{0}, \cdots, v_{m+q+1}\right\}$, respectively. 
The hull forms can be expressed parametrically by using the form parameters to constitute the feature curves and hull section frames. The general procedure to generate fairing surfaces of the hull form observes four steps, as presented in Figure 4. First, the form parameters should be suitably selected. Second, the parametric design of the longitudinal feature curves is performed. Third, transversal feature curves can be similarly generated and a set of surfaces can be derived by means of interpolation. Finally, fairing form variations can be obtained [23]. It should be noted that the number and the range of the form parameters should be selected carefully to prevent any distortion of the ship. In the study, eight form parameters are selected for the generation of the bulbous bow. More details about these parameters are presented in the Example Study section.

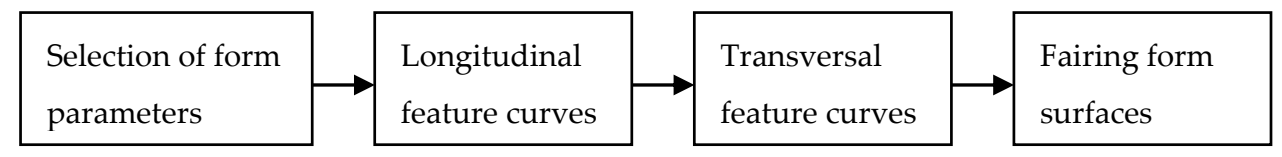

Figure 4. Parametric modeling of a hull form.

\section{Calculation of Wave-Making Resistance}

In the study, the optimization objective is to minimize the wave-making resistance. Suppose the fluid is inviscid and incompressible, and Rankine-source panel method is employed.

For a ship traveling with a steady forward speed $U$ in the calm water of infinite depth, the wave-making velocity potential $\varphi(x, y, z)$ satisfies the following equations:

$$
\begin{gathered}
\text { in the fluid domain : } \nabla^{2} \phi=0 \\
\text { on the free surface }:=\frac{1}{g}\left(-U_{s} \cdot \nabla \phi+\frac{1}{2} \nabla \phi \cdot \nabla \phi\right) \\
\text { on the free surface: }\left(\nabla \phi-U_{s} \cdot \vec{n}\right) \cdot \nabla \xi=\phi_{z} \\
\text { on the hull surface : } \frac{\partial \phi}{\partial n}=U_{s} \cdot \vec{n} \\
\text { at infinity: } \quad \nabla \phi=(0,0,0)
\end{gathered}
$$

where $U_{s}=(U, 0,0), \mathrm{g}$ is the acceleration of gravity, and $\xi$ is the free surface elevation.

The velocity potential at the field point $P$ can be calculated as [25]

$$
\phi(P)=-\frac{1}{4 \pi} \iint_{S B} \sigma(Q)\left(\frac{1}{r(P, Q)}+\frac{1}{r^{\prime}\left(P, Q^{\prime}\right)}\right) d s-\frac{1}{4 \pi} \iint_{S F} \sigma(Q)\left(\frac{1}{r(P, Q)}+\frac{1}{r^{\prime}\left(P, Q^{\prime}\right)}\right) d s
$$

where $Q$ and $Q^{\prime}$ are source points, $\sigma(Q)$ is the source strength, and $r$ and $r^{\prime}$ are the distances between the field point and source points.

When the ship is sailing, the wave is steep and the floating state cannot be neglected. Therefore, the nonlinear free surface boundary condition should be used. The method of Taylor series expansion is applied to Equations (4) and (5) by making use of the approximate solution $z=Z$ and $\varphi=\phi$. Then, the boundary condition on the approximate free surface can be obtained as:

$$
\begin{gathered}
{\left[2\left(\nabla A-U \nabla \varphi_{1}\right)+\vec{W} B\right] \cdot \nabla \phi+\vec{W} \cdot[(\vec{W} \cdot \nabla) \nabla \varphi]+g \phi_{z}=2 \nabla \varphi \cdot\left[\nabla A-U \nabla \varphi_{1}\right]+B(A-g Z)} \\
\xi=Z+\frac{\vec{W} \cdot \nabla \phi-A+g Z}{\vec{W} \cdot \nabla \varphi_{z}-g}
\end{gathered}
$$


where $\vec{W}=\nabla \phi-U_{s}, A=\frac{1}{2} \nabla \varphi \cdot \nabla \varphi$ and $B=\frac{\left[\vec{W} \cdot\left(\nabla A-U \nabla \varphi_{x}\right)+g \varphi_{z}\right]}{\vec{W} \cdot \nabla \varphi_{z}-g}$.

The boundary condition on the ship surface is:

$$
\nabla \phi \cdot \vec{n}=U n_{1}
$$

Based on Bernoulli equation, the pressure can be calculated as

$$
p=\rho\left(U \phi_{x}-(\nabla \phi \cdot \nabla \phi) / 2+g z\right)
$$

where $U$ is the ship velocity, and $\rho$ is the fluid density. By integrating the pressure along the wetted boundary surface, one has the hydrodynamic force on the hull as

$$
\vec{F}=\left(F_{1}, F_{2}, F_{3}\right)=\iint_{S B} p \vec{n} d S
$$

Thus, the wave-making resistance and its nondimensional form can be obtained as

$$
R_{w}=-F_{1}=-\iint_{S B} p n_{1} d S
$$

and

$$
C_{w}=\frac{R_{w}}{0.5 \rho U^{2} S_{0}}
$$

respectively, where $S_{0}$ is the wetted surface area in calm waters. $C_{w}$ is also named as the coefficient of wave-making resistance.

\section{Optimization Algorithms}

To guarantee the globally optimal solution and solving efficiency as well, a hybrid algorithm is applied in the study. Ensemble Investigation is employed to obtain the globally optimal solution. This algorithm allows permuting a set of design variables and generating DOE (design of experiments) tables, which is very effective to gather information about the optimization problem investigated in the whole design space. Generated DOE tables are useful to detect the trends of the optimization variables with regard to the optimization objectives, irrespective of constraints [26]. This optimization approach is performed by creating several different versions of a proposed new design, and then selecting the option that best fits the design goals. The generation of a systematic series of design options can be completed soon. These options are tested against design goals-for example, the hydrodynamic performance. Computational techniques such as regression analysis can be used to determine the "best" option.

To improve the efficiency of solution-searching, the T-Search algorithm is incorporated. This algorithm is a method of feasible direction. It uses a direct search until some constraint gets violated. At the expense of a little extra effort, T-Search is characterized by efficient operation within the admissible region with satisfactory performance along the boundary of that region [27]. The other two features of the algorithm are the allowance made for small perturbations of the base point and the flexible directions for exploration in the tangent hyperspace [28]. It consists of exploratory moves that start from a so-called base point along the variable axes followed by global moves in the descent search direction found in successful exploratory moves. If a constraint bound is approached, then it returns to the last admissible point and performs a unidirectional optimization along the hyperplane tangent to the active constraint either to keep the search in the feasible domain or to bring it back to the feasible domain. T-Search is able to find the local minimum of an arbitrary, explicitly-stated function of more than one variable, subject to arbitrary, non-linear constraints. 


\section{Optimization Scheme}

\subsection{Optimization Goal and Design Variables}

The optimization objective in the study is to minimize the wave-making resistance under designed ship velocity, i.e., $\min \left\{C_{w}\right\}$. The constraints are set to be subject to (1) $\left|\Delta_{0}-\Delta_{p}\right| / \Delta_{0} \leq 1 \%$ where $\Delta_{0}$ is the initial displacement, and $\Delta_{p}$ is the displacement of optimized ship; (2) $\left|L C B_{0}-L C B_{p}\right| / L C B_{0} \leq 1 \%$, where $L C B_{0}$ is the initial longitudal coordiante of the boyance center while $L C B_{p}$ is the longitudal coordiante of boyance center of the optimized ship; and (3) the ship length, breadth and draft are constant during the optimization process.

Since the lines of the bulbous bow are vital to the wave-making resistance, the design variables are selected as form control parameters of bulbous bow, including the distance between the center of the bulb bow and the front end of the bulb bow $\left(D_{c f}\right)$; the height of the front end of the bulb bow $\left(H_{f}\right)$; the contour fullness of the top longtitudinal section of bulb bow $\left(F_{t l}\right)$ and its coefficient $\left(C_{f t l}\right)$; the contour fullness of the low longtitudinal section of bulb bow $\left(F_{l l}\right)$ and its coefficient $\left(C_{f l l}\right)$; the contour fullness at the half beam of the bulb bow $\left(F_{h b}\right)$; and half of the maximal breadth of the bulb bow $\left(B_{h}\right)$. These form control parameters are used to generate the basic feature curves of the bulbous bow, as shown in Figure 2.

\subsection{Optimization Framework}

In the optimization framework, there are mainly three modules: the parametric modeling, CFD calculation and lines modification. The optimization operation is performed in the SHIPFLOW-CAESES DESIGN environment. First, NURBS based parametric modeling is performed in the CAESES and the lines offsets of the given hull can be obtained. Then, the files of the lines of offsets are automatically imported into the SHIPFLOW so that the CFD calculation can be carried out. Afterwards, the CFD results are imported to CAESES as comparison with the optimization objective. If the optimization goal is achieved, the lines are exported as the optimal lines; otherwise, the design variables are modified based on the hybrid optimization algorithm, and the optimization operation is repeated until the optimization goal is achieved. For general procedure, the optimization design is presented in Figure 5.

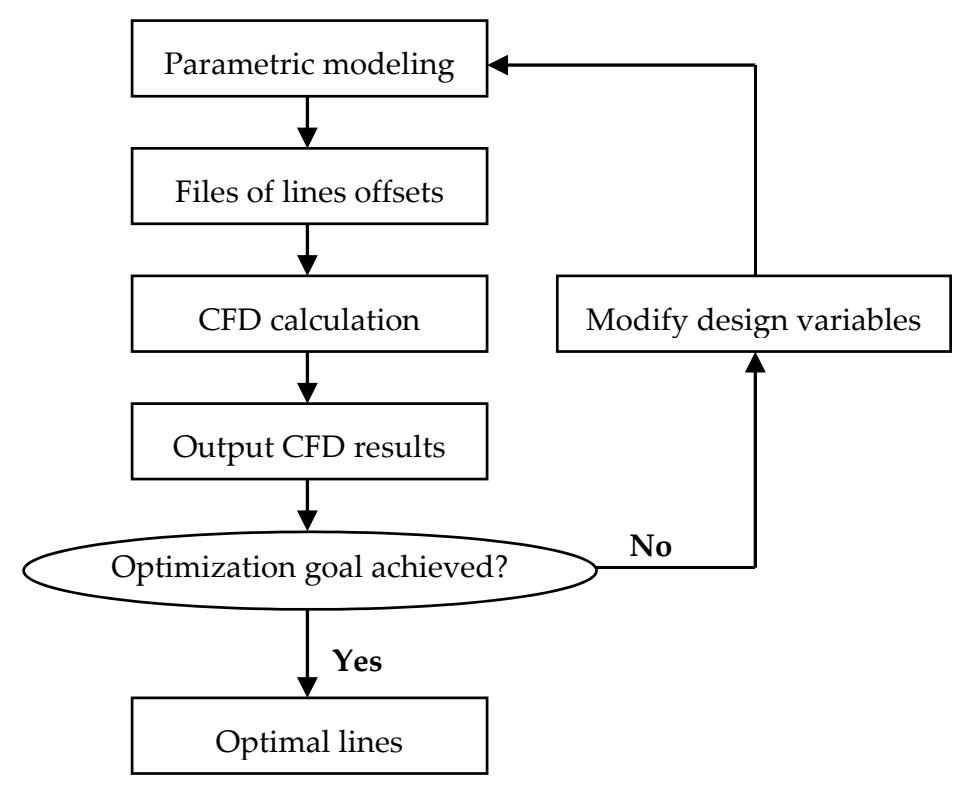

Figure 5. Optimization procedure. 
It is noted that a batch file is necessary to connect SHIPFLOW and CAESES applications. SHIPFLOW provides the batch file in the installation package. The batch file includes echo commands, pause commands, call commands, start commands, go-to commands, and set commands. There is a boundary of surface between the surface of the bulbous bow and the surface of the front part of hull. This boundary is maintained between optimization circles so that the bulbous bow can connect to the front part of hull smoothly.

\section{Example Study}

A Ro-Ro ship is investigated to verify the optimization scheme proposed in the study. The main particulars of the ship are listed in Table 2 . The ship velocity is 22 knots. In terms of Froude number, one has $F r=0.28$.

Table 2. Main particulars of a Ro-Ro ship.

\begin{tabular}{ccc}
\hline Description & Variable & Value \\
\hline Length $(\mathrm{m})$ & $L$ & 165 \\
Breadth $(\mathrm{m})$ & $B$ & 24.8 \\
Draft $(\mathrm{m})$ & $d$ & 8.7 \\
Block coefficient & $C_{b}$ & 0.65 \\
\hline
\end{tabular}

In the CAESES framework, the form parameters are imported to the curve generator to obtain a set of feature curves. Then, fairing surfaces can be derived automatically through a surface generator. The parameterized hull surfaces are shown in Figure 6.

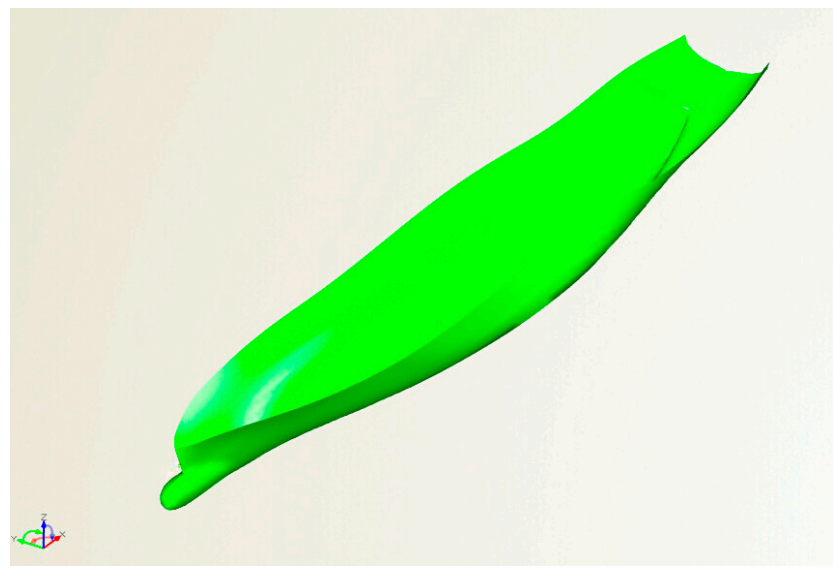

Figure 6. Hull surfaces of the Ro-Ro ship.

Based on the optimization framework shown in Figure 5, the hybrid optimization algorithm is performed. In detail, the Ensemble Investigation is employed to obtain feasible solutions in the whole design space. A set of optimized hull forms that satisfy the constraints are derived. Then, the T-Search algorithm is used within the set to determine the solution with minimal wave-making resistance. The design variables and their variations are listed in Table 3. The results of optimization objective and constraints are presented in Table 4. 
Table 3. Optimization results of the design variables.

\begin{tabular}{cccc}
\hline Design Variable & Range & Initial Value & Optimized Value \\
\hline$D_{c f}(\mathrm{~m})$ & {$[0,1]$} & 0.624 & 0.237 \\
$H_{f}(\mathrm{~m})$ & {$[1,5.5]$} & 4.287 & 4.856 \\
$F_{t l}$ & {$[0.1,0.6]$} & 0.41 & 0.34 \\
$C_{f t l}$ & {$[0.52,0.91]$} & 0.73 & 0.842 \\
$F_{l l}$ & {$[1.12,1.37]$} & 1.28 & 1.31 \\
$C_{f l l}$ & {$[0.65,0.96]$} & 0.85 & 0.943 \\
$F_{h b}$ & {$[1.4,2.0]$} & 1.64 & 1.873 \\
$B_{h}(\mathrm{~m})$ & {$[2.1,2.58]$} & 2.301 & 2.384 \\
\hline
\end{tabular}

$D_{c f}$, the distance between the center of the bulb bow and the front end of the bulb bow; $H_{f}$, the height of the front end of the bulb bow; $F_{t l}$, the contour fullness of the top longtitudinal section of the bulb bow; $C_{f t l}$, the coefficient of the contour fullness of the top longtitudinal section; $F_{l l}$, the contour fullness of the low longtitudinal section of the bulb bow; $C_{f l l}$, the coefficient of the contour fullness of the low longtitudinal section; $F_{h b}$, the contour fullness at the half beam of the bulb bow; $B_{h}$, half of the maximal breadth of the bulb bow.

Table 4. Optimization results of the objective and constraints.

\begin{tabular}{cccc}
\hline Description & Initial Value & Optimized Value & Variation \\
\hline Nondimensional wave-making resistance, $C_{w}$ & 0.0020368 & 0.0019488 & $4.32 \%(\downarrow)$ \\
Displacement, $\Delta(\mathrm{t})$ & 22357.695 & 22382.706 & $0.112 \%(\uparrow)$ \\
Longitudal coordiante of boyance center, $L C B(\mathrm{~m})$ & 0.515798 & 0.515262 & $0.104 \%(\downarrow)$ \\
\hline
\end{tabular}

As can be seen from the optimization results, the wave-making resistance is reduced by modifying the design variables while the constraints are satisfied. The initial values of design variables are one set of the feasible solutions family. The variation of the design variables indicates that smaller $D_{c f}$ and $F_{t l}$, against larger $H_{f}, C_{f t l}, F_{l l}, C_{f l l}, F_{h b}$, and $B_{h}$ help reduce the wave-making resistance. The comparison of the wave contours on free surfaces before and after optimization is presented in Figure 7. The comparison of the longitudinal wave cuts before and after optimization is given as Figure 8. The right plot in Figure 7 presents the changes of wave contours on the forward part of the ship. Similarly, in Figure 8, the right plot highlights the changes of wave cuts on the area around the bulbous bow.
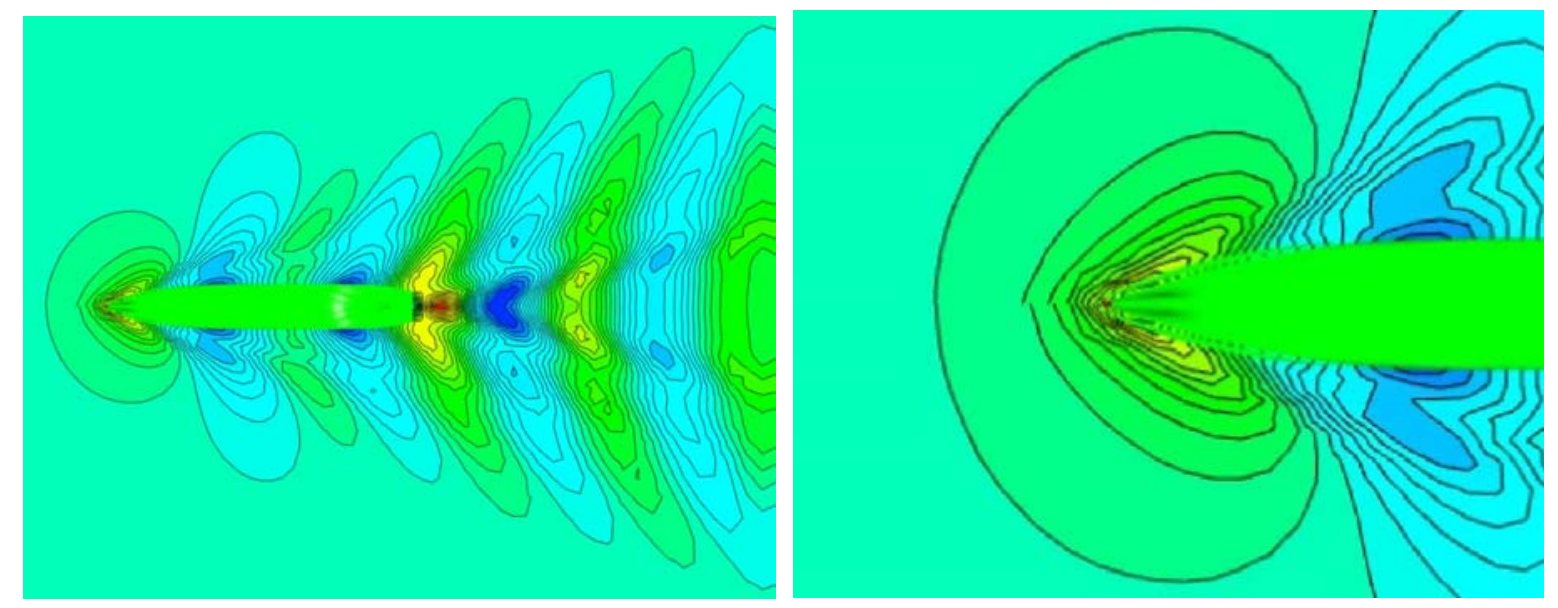

Figure 7. Comparison of the wave contours on free surfaces before and after optimization; upper part,-before optimization; lower part,-after optimization.

As can be seen from the wave variation, the wave numbers and the wave amplitude decrease obviously around the bow after the lines of the bulb bow are optimized, which implies a decrease of wave-making resistance. Figures 9 and 10 present the variation of the lines of the bulb bow. 
In Figure 9, the left plot is the original form of the bulb bow, while the right plot is the optimized form. In Figure 10, the yellow lines represent the optimized results while the black lines are with the original form. From the comparison results, it can be indicated that a stretch of the bulb bow upwards helps reduce the wave-making resistance.
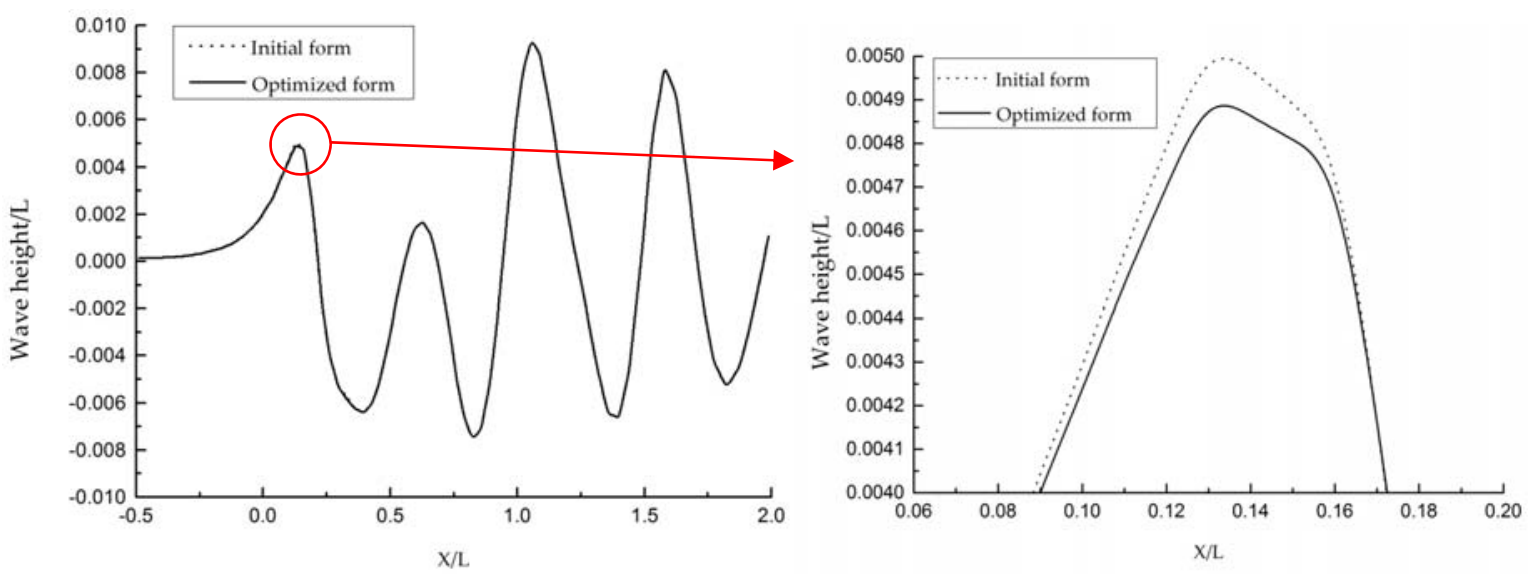

Figure 8. Comparison of the longitudinal wave cuts before and after optimization $(y / L=0.1)$. $\mathrm{L}$, ship length; units in $\mathrm{m} / \mathrm{m}$.
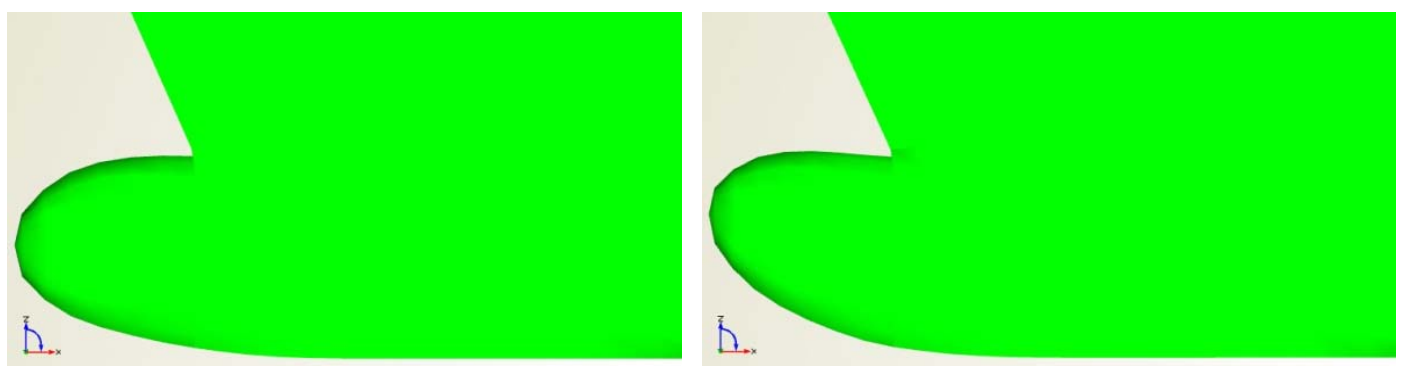

Figure 9. Comparison of the bulbous bows before and after optimization: left,-original; right,-optimized.

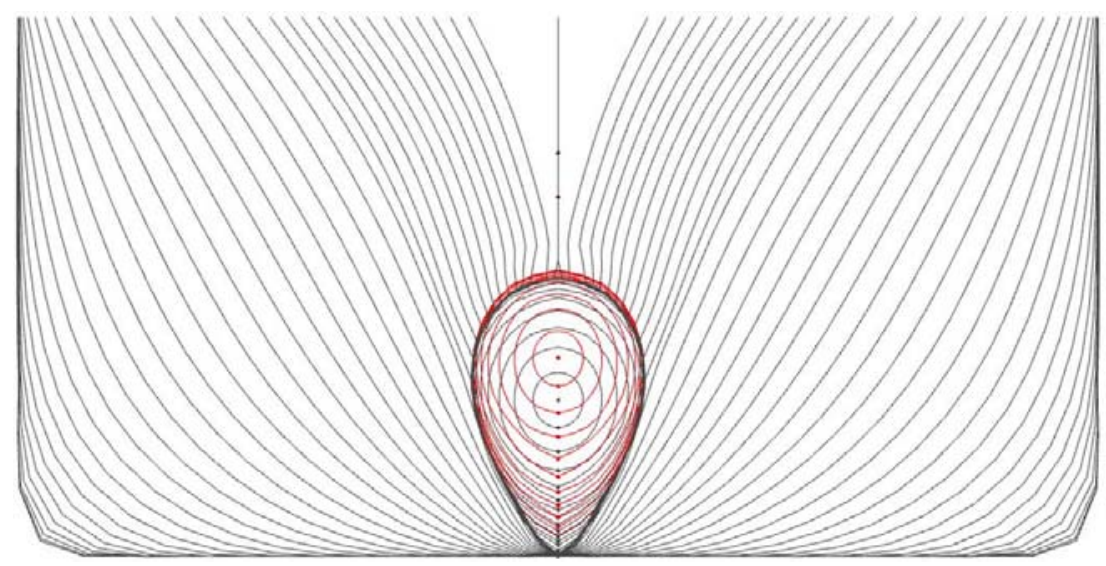

Figure 10. Comparison of the lines before and after optimization.

\section{Conclusions}

The combination of CFD and CAD improves the quality of ship hull form design. In this paper, an optimization design strategy is proposed based on SHIPFLOW-CAESES framework. The optimization results demonstrate the feasibility of using successfully different software systems in 
the optimization process. A fully automatic optimization integration framework is achieved by using CAESES coupled with CFD solver SHIPFLOW. Through the framework, the full parametric modeling of the hull, hydrodynamic calculation, design evaluation and shape variation are implemented effectively, which improves the quality and efficiency of ship design. The NURBS method is employed in the parametric modeling of the hull forms, while potential theory is used to calculate the wave-making resistance of the ship hull. To improve the optimization efficiency, a hybrid optimization scheme is taken. The validity of the proposed optimization design framework is confirmed by applying the optimization framework to a Ro-Ro ship. Based on the simulation results, some recommendations can be summarized as follows: (i) the smaller $D_{c f}$ and $F_{t l}$, against larger $H_{f}, C_{f t l}, F_{l l}, C_{f l l}, F_{h b}$, and $B_{h}$ helps reduce the wave-making resistance; and (ii) a stretch of the bulb bow upwards also helps reduce the wave-making resistance.

In this study, only the bulbous bow optimization is concerned. In the next work, the optimization of the entire hull will be studied. Moreover, total resistance will be considered in the optimization design, instead of the only wave-making resistance. In addition, another calculation method for wave resistance, i.e., the wave cut analysis will be studied. As mentioned in literature, wave cut analysis provides a more accurate and robust solution. Moreover, for a planning hull, instead of the displacement hull that is investigated in the study, wave cut analysis might be preferable.

Acknowledgments: This work was partially supported by the Special Item supported by the Fujian Provincial Department of Ocean and Fisheries (No. MHGX-16), and the Special Item for the University in Fujian Province supported by the Education Department (No. JK15003).

Author Contributions: Weilin Luo and Linqiang Lan analyzed the data; Weilin Luo wrote the paper.

Conflicts of Interest: The authors declare no conflict of interest.

\section{References}

1. Yusuke, T.; Sugimoto, S.; Shinya, M. Development of CAD/CFD/optimizer-integrated hull-form design system. J. Kansai Soc. Naval Archit. 2003, 240, 29-36.

2. Ahmadzadehtalatapeh, M.; Mousavi, M. A review on the drag reduction methods of the ship hulls for improving the hydrodynamic performance. Int. J. Marit. Technol. 2015, 4, 51-64.

3. Percival, S.; Hendix, D.; Noblesse, F. Hydrodynamic optimization of ship hull forms. Appl. Ocean Res. 2001, 23, 337-355. [CrossRef]

4. Tahara, Y.; Tohyama, S.; Katsui, T. CFD-based multi-objective optimization method for ship design. Int. J. Numer. Methods Fluids 2006, 52, 499-527. [CrossRef]

5. Abt, C.; Harries, S. Friendship-Framework-Integrating ship-design modelling, simulation, and optimization. Naval Archit. 2007, 1, 36-37.

6. Harries, S.; Abt, C. Tight and loose coupling of CAD and CFD-Methods, practical aspects, advantages. In Proceedings of the RINA International Conference-Marine CFD 2008, Southampton, UK, 26-27 March 2008; pp. 35-41.

7. Couser, P.; Harries, S.; Tillig, F. On design-space exploration and design refinement by numerical simulation. In Proceedings of the RINA International Conference in Marine Design, Coventry, UK, 14-15 September 2011; pp. 101-110.

8. Brizzolara, S.; Vernengo, G. Automatic computer driven optimization of innovative hull forms for marine vehicles. In Proceedings of the 10th WSEAS International Conference on Applied Computer and Applied Computational Science, ACACOS'11, Venice, Italy, 8-10 March 2011; pp. 273-278.

9. Biliotti, I.; Brizzolara, S.; Viviani, M.; Vernengo, G.; Ruscelli, D.; Galliussi, M.; Guadalupi, D.; Manfredini, A. Automatic parametric hull form optimization of fast naval vessels. In Proceedings of the 11th International Conference on Fast Sea Transportation, FAST 2011, Honolulu, HI, USA, 26-29 September 2011; pp. 294-301.

10. Ginnis, A.I.; Feurer, C.; Belibassakis, K.A.; Kaklis, P.D.; Kostas, K.V.; Gerostathis, T.P.; Politis, C.G. A CATIA ${ }^{\circledR}$ ship-parametric model for isogeometric hull optimization with respect to wave resistance. In Proceedings of the International Conference on Computer Applications in Shipbuilding, Trieste, Italy, 20-22 September 2011. 
11. Brenner, M.; Zagkas, V.; Harries, S.; Stein, T. Optimization using viscous flow computations for retrofitting ships in operation. In Proceedings of the 5th International Conference on Computational Methods in Marine Engineering, MARINE 2013, Hamburg, Germany, 29-31 May 2013; pp. 69-80.

12. Zakerdoost, H.; Ghassemi, H.; Ghiasi, M. An evolutionary optimization technique applied to resistance reduction of the ship hull form. J. Naval Archit. Mar. Eng. 2013, 10, 1-12. [CrossRef]

13. Vernengo, G.; Brizzolara, S.; Bruzzone, D. Resistance and seakeeping optimization of a fast multihull passenger ferry. Int. J. Offshore Polar Eng. 2015, 25, 26-34.

14. Brizzolara, S.; Vernengo, G.; Pasquinucci, C.A.; Harries, S. Significance of parametric hull form definition on hydrodynamic performance optimization. In Proceedings of the MARINE 2015-Computational Methods in Marine Engineering VI, Rome, Italy, 15-17 June 2015; pp. 254-265.

15. Ang, J.; Goh, C.; Li, Y. Hull form design optimization for improved efficiency and hydrodynamic performance of "ship-shaped" offshore vessels. In Proceedings of the International Conference on Computer Applications in Shipbuilding, Bremen, Germany, 29 September-1 October 2015; pp. 1-10.

16. Wang, J. A NURBS-Based Computational Tool for Hydrodynamic Optimization of Ship Hull Forms. Ph.D. Thesis, George Mason University, Fairfax, VA, USA, 2015.

17. Tahara, Y.; Stern, F.; Himeno, Y. CFD-based optimization of a surface combatant. J. Ship Res. 2004, 48, 273-287.

18. Campana, E.F.; Peri, D.; Pinto, A.; Stern, F.; Tahara, Y. A comparison of global optimization methods with application to ship design. In Proceedings of the 5th Osaka Colloquium on Advanced Research on Ship Viscous Flow and Hull Form Design by EFD and CFD Approaches, Osaka, Japan, 14-15 March 2005.

19. Wen, A.S.; Mariyam, S.; Shamsuddin, H.; Samian, Y. Optimized NURBS ship hull fitting using simulated annealing. In Proceedings of the International Conference on Computer Graphics, Imaging and Visualisation, Sydney, Australia, 26-28 July 2006; pp. 484-489.

20. Pinto, A.; Peri, D.; Campana, E.F. Multiobjective optimization of a containership using deterministic particle swarm optimization. J. Ship Res. 2007, 51, 217-228.

21. Ayob, M.; Ahmad, F.; Ray, T.; Smith, W. A hydrodynamic preliminary design optimization framework for high speed planing craft. J. Ship Res. 2012, 56, 35-47. [CrossRef]

22. Takai, T. Simulation Based Design for High Speed Sea Lift with Waterjets by High Fidelity URANS Approach. Master's Thesis, University of Iowa, Iowa City, IA, USA, 2010.

23. Han, S.; Lee, Y.; Choi, Y.B. Hydrodynamic hull form optimization using parametric models. J. Mar. Sci. Technol. 2012, 17, 1-17. [CrossRef]

24. Zhang, P.; Zhu, D.; He, S. The parametric design method of hull form. Ship Build. China 2008, 49, $26-34$.

25. Liu, Y. Theory of Ship Wave Making Resistance; National Defense Industry Press: Beijing, China, 2003.

26. Koutroukis, G. Parametric Design and Multi-Objective Optimization-Study of an Ellipsoidal Containership. Master's Thesis, National Technical University of Athens, Athens, Greece, 2012.

27. Save, M.; Prager, W. Structural Optimization: Volume 2: Mathematical Programming; Springer: New York, NY, USA, 1990.

28. Hilleary, R.R. The Tangent Search Method of Constrained Minimization; U.S. Naval Postgraduate School: Monterey, CA, USA, 1966.

(C) 2016 by the authors; licensee MDPI, Basel, Switzerland. This article is an open access article distributed under the terms and conditions of the Creative Commons Attribution (CC-BY) license (http://creativecommons.org/licenses/by/4.0/). 